\title{
Switching Faraday rotation on a molecular level
}

\author{
Stefaan Vandendriessche, Ward Brullot and Thierry Verbiest \\ Laboratory for Molecular Imaging and Photonics, \\ Katholieke Universiteit Leuven, Celestijnenlaan 200 D, B-3001 Heverlee, Belgium
}

\begin{abstract}
In this work we present measurements of the switching of the Faraday effect in metal-organic compounds. Faraday rotation is the rotation of the plane of polarization of linearly polarized light under the influence of a magnetic field in the direction of propagation of the light. It is the magnetic equivalent of circular birefringence and is related to magnetic circular dichroism via the Kramers-Kronig transformation. The Faraday effect is used in optical isolators and magnetic sensors.

Faraday rotation and magnetic circular dichroism spectra have been calculated and measured for various nanoparticles, nanocomposites, magnetic fluids and metal-organic complexes. These measurements and calculations indicate that it is possible to change the magneto-optical response by changing the state of the molecule, such as a change in protonation or oxidation state. The molecular environment also influences the magnetooptical spectra of metal-organic complexes and organic molecules. Thus it is possible to change the Faraday rotation spectrum by modifying the molecular environment or the molecule itself. We have measured the reversible switching of the magneto-optical response by these principles. This easily induced reversible switching opens the possibility of new devices such as switchable optical isolators.
\end{abstract}

Keywords: Faraday rotation

\section{INTRODUCTION}

Faraday rotation is the rotation of the polarization of light due to magnetically induced circular birefringence, induced by a magnetic field parallel to the propagation of the light. It was discovered by M. Faraday, ${ }^{1}$ and can be described by the formula

$$
\theta=V B L
$$

Where $\theta$ is the rotation of polarization, $B$ is the amplitude of the magnetic field parallel to the propagation of light, $L$ is the length of propagation through the magnetic field and $V$ is the Verdet constant expressed in deg. $\mathrm{T}^{-1} \mathrm{~m}^{-1}$, a material parameter.

Faraday rotation differs from naturally ocurring optical rotation in a key aspect: non-reciprocality. Upon reflection through a sample, natural optical rotation is canceled because it is reciprocal. Faraday rotation on the other hand is non-reciprocal, and as such rotation accumulates upon multiple reflections through a sample. This advantage is important in the associated applications, and is a very useful feature for the measurement of Faraday rotation. By measuring the Faraday rotation after an even number of reflections through a sample, reciprocal effects cancel out, leaving only non-reciprocal effects such as Faraday rotation. Additionally, these effects are amplified in magnitude by the number of times light has traveled through the sample, facilitating the measurement and application of these effects.

Faraday rotation is employed in a broad range of applications such as optical isolators, ${ }^{2}$ magnetic field sensors ${ }^{3}{ }^{\text {current sensors }}{ }^{4}$ and displacement sensors. ${ }^{5}$ It can also be used to acquire information on the structure of magnetic materials, ${ }^{6}$ quantum $\operatorname{dots}^{7}$ and semiconductors ${ }^{8}$ or to derive information about the structure of the universe ${ }^{9}$ this is possible due to the relative simplicity yet high precision of the measurement of Faraday rotation. ${ }^{10}$ All of this knowledge and these applications rely on knowledge of the Verdet constant.

The Verdet constant, $V$, is a wavelength dependent material parameter. Organic materials have relatively small Verdet constants in regions far from resonance, typically hundreds of degrees per Tesla per meter in the

Send correspondence to stefaan.vandendriessche@fys.kuleuven.be

Nonlinear Optics and Applications VI, edited by Benjamin J. Eggleton, Alexander L Gaeta, Neil G. Broderick, Proc. of SPIE Vol. 8434, 84341E - @ 2012 SPIE · CCC code: 0277-786X/12/\$18 - doi: 10.1117/12.921856 
visible wavelength region, displaying a quadratic decline towards longer wavelengths. ${ }^{11-14}$ In contrast to typically diamagnetic organic materials, para- and ferromagnetic inorganic materials such as iron oxide films, ferrofluids ${ }^{15}$ or iron garnet films ${ }^{16}$ display Verdet constants in the visible wavelength region on the order of millions of degrees per Tesla per meter.

Magnetic circular dichroism (MCD) is a difference in absorption between left- and right-hand circularly polarized light due to a magnetic field parallel to the direction of the propagation of light. While natural circular dichroism (NCD) requires a lack of inversion centers and mirror planes at a molecular level, MCD does not have these requirements and can be present in all materials. It is mainly used for characterization, providing an excellent fingerprinting capability. The main advantages of MCD compared to standard absorption spectroscopy are the bisignate nature of MCD and the more stringent selection rules, which provide greatly enhanced resolution. ${ }^{17}$ The disadvantages are that the material must contain magnetically active components. A recent example of MCD in analysis is the use of MCD to analyze quantum effects in single molecule magnets. ${ }^{18}$ Additionally it is frequently used to characterize and analyze metallo-proteins, amongst others providing indispensible information on the spin and oxidation states of heme groups. ${ }^{17}$

In this work we present measurements of the reversible switching of the magneto-optical response in solution. These data can be extended to further research on electro-chemical switching of magneto-optical response. This allows for novel, reversibly switchable magneto-optical devices.

\section{EXPERIMENTAL DETAILS}

The investigated solutions were made by dissolving $\mathrm{CoCl}_{2} \cdot 6 \mathrm{H}_{2} \mathrm{O}$, purchased from Sigma Aldrich, in MilliQ water. Acidification of the solution was achieved by adding $37 \mathrm{~m} \% \mathrm{HCl}$ solution, and neutralization was achieved by adding $\mathrm{KOH}$ solution.

In order to spectrally measure both Faraday rotation and MCD, a laser driven light source (LDLS) was employed (Energetiq LDLS EQ-99). The generated light was focussed into a Cornerstone 260 1/4 m monochromator which was operated by a computer. The slits were chosen such that the resulting beam had a full width half maximum of approximately $2 \mathrm{~nm}$. Subsequently the collimated beam was sent through a linear polarizer, followed by a photo-elastic modulator (PEM) with its axis oriented at $45^{\circ}$ respective to the polarization of the light. The light then passed through a $2 \mathrm{~mm}$ optical quartz cuvette in a 1 Tesla DC magnet with magnetic field in the direction of the propagation of light. After passing through a polarizer which polarized the light perpendicular to the axis of the PEM, the light was collected by a photomultiplier tube (PMT). The electrical signal generated by the PMT was fed into two separate lock-in amplifiers. The operating frequency (approximately 50 $\mathrm{kHz}$ ) of the PEM served as reference frequency in the lock-in amplifier (Stanford Research Systems SR830) for the circular dichroism measurements, and the second harmonic of the operating frequency served as the reference frequency in a second lock-in amplifier for the optical rotation measurements. The measured lock-in values were read in by a personal computer.

In order to analyze the resulting signal, it is necessary to analyze the Mueller matrices of the optical components. The derivation is beyond the scope of this paper but can be summarized by the fact that the signal at the reference frequency of the PEM is proportional to the circular dichroism present in the sample while the signal at the second harmonic of the PEM reference frequency is proportional to the optical rotation. By measuring both values for multiple discrete magnetic fields it is possible to extract the magnetic circular dichroism and the Faraday rotation. ${ }^{19}$ Dividing the Faraday rotation by the pathlength of the sample results in the Verdet constant.

With knowledge of either the Faraday or MCD spectrum of a material, it is possible to use the KramersKronig relations in order to calculate the other spectrum. ${ }^{20}$ This is only exact if one of the two magneto-optical spectra is known from $0 \rightarrow \infty \mathrm{nm}$, which is not the case here; as such both spectra were measured. Despite this it is possible to obtain qualitatively satisfactory spectra using correct boundary conditions. 


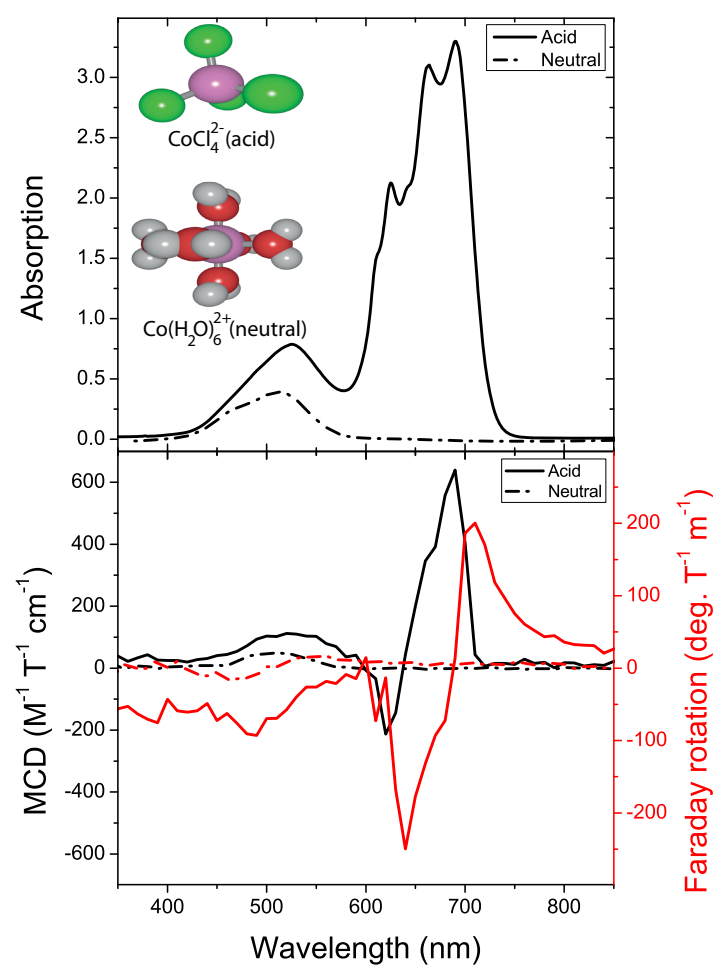

Figure 1. Absorption, $\mathrm{MCD}$ and Faraday rotation spectra of a $0.42 \mathrm{M}$ aqeuous solution of $\mathrm{CoCl}_{2} \cdot 6 \mathrm{H}_{2} \mathrm{O}$. $\mathrm{Neutral}$ is a solution in MilliQ water and Acid is a solution in $18.5 \mathrm{~m} \% \mathrm{HCl}$.

\section{RESULTS - TODO MOLAR ELIPTICITY UNITS}

Figure 1 displays the absorption, MCD and Faraday rotation spectra of a $0.42 \mathrm{M}$ aqueuos acid and neutral solution of $\mathrm{CoCl}_{2} \cdot 6 \mathrm{H}_{2} \mathrm{O}$ from $350 \mathrm{~nm}$ to $850 \mathrm{~nm}$. These spectra respectively correspond to the spectra of the $\mathrm{CoCl}_{4}^{2-}$ ion and the $\mathrm{Co}\left(\mathrm{H}_{2} \mathrm{O}\right)_{6}^{2+}$ ion. While both of these ions are $\mathrm{d} 7$ systems, the first is present in a tetrahedral configuration and the second is a present as an octahedral complex. Despite the fact that the $\mathrm{CoCl}_{4}^{2-}$ ion is distorted from a perfect tetrahedral configuration, the resulting splitting is sufficiently small to neglect in the further analysis of the spectrum. ${ }^{21}$

Four clear bands can be seen in the absorption spectrum of the solution acidified by addition of concentrated $\mathrm{HCl}$, with another band appearing as a slight shoulder on the stronger bands at approximately $605 \mathrm{~nm}$. While the strong band at approximately $525 \mathrm{~nm}$ is present in both complexes, all the other bands disappear upon complexation with water. ${ }^{22}$

In order to explain the MCD spectrum of the $\mathrm{CoCl}_{4}^{2-}$ complex, it is necessary to analyze the energy diagram of the complex. This energy diagram is depicted in Figure 2.

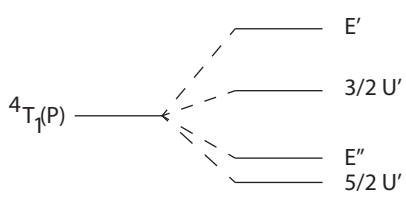

Figure 2. Energy level diagram for $\mathrm{CoCl}_{4}^{2-}$. 


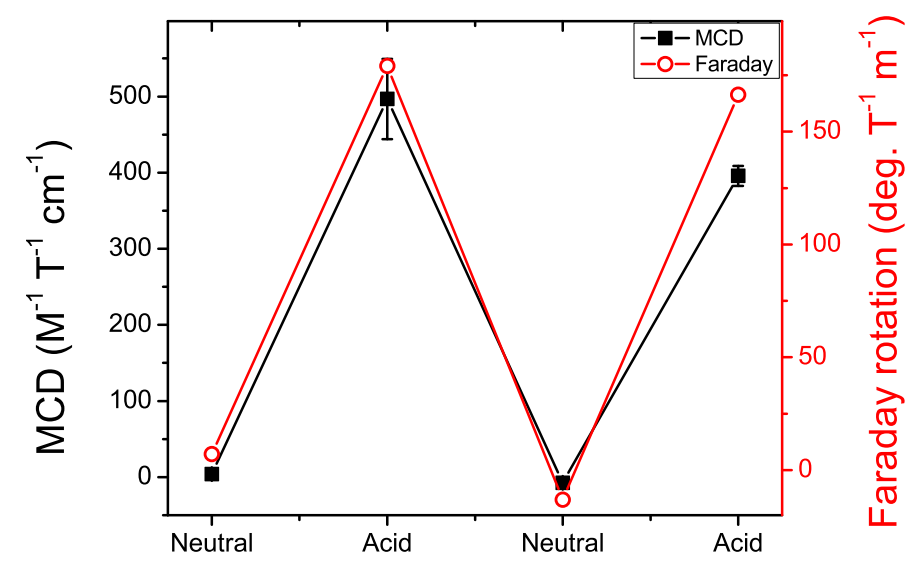

Figure 3. Demonstration of the reversible switching of Faraday rotation and MCD for a $\mathrm{CoCl}_{2} \cdot 6 \mathrm{H}_{2} \mathrm{O}$ solution. Left axis is the MCD at $690 \mathrm{~nm}$ and right axis is the Faraday rotation at $710 \mathrm{~nm}$. The sample was switched from neutral to acid twice with concentrated $\mathrm{HCl}$, returning to the original Faraday rotation and MCD values.

The observed MCD transitions, besides the band at $525 \mathrm{~nm}$, can all be assigned to transitions to ${ }^{4} \mathrm{~T}_{1} \mathrm{P}$ in the $\mathrm{CoCl}_{4}^{2-}$ complex. The band at $690 \mathrm{~nm}$ corresponds to the $5 / 2 \mathrm{U}$ ' transition and the band at $660 \mathrm{~nm}$ to the E" transition. The band at $620 \mathrm{~nm}$ corresponds to both the $3 / 2 \mathrm{U}$ ' and the E' transition, which can not be resolved with the employed experimental setup. Most of the measured MCD originates from a $\mathrm{C}$ term ${ }^{23}$ which is associated with a degenerate ground state without spin-orbit coupling and an excited state with spin-orbit coupling.

The observed Faraday rotation can be ascribed to the same transitions that cause the MCD. However, the intensities of the transition are different, and can not be accurately predicted by using the pure crystal field theory employed to explain the MCD spectrum. ${ }^{24}$ To fully explain the intensities it is necessary to include molecular orbital theory, which is beyond the scope of this paper which demonstrates preliminary results on the ability to switch the magneto-optical response of materials.

Finally, with the obtained understanding of the (magneto-optical) spectra of both complexes, it is possible to exploit the differences in their spectra for the purpose of switching magneto-optical response. This is demonstrated in Figure 3.

By changing the concentration of $\mathrm{HCl}$ in the solution, it is possible to change the predominant complex present. This changes the magneto-optical response of the solution correspondingly. The $\mathrm{CoCl}_{4}^{2-}$ complex shows strong magneto-optical response in the $690 \mathrm{~nm}$ to $710 \mathrm{~nm}$ range, while the $\mathrm{Co}\left(\mathrm{H}_{2} \mathrm{O}\right)_{6}^{2+}$ complex displays no magneto-optical response in this region. Addition of concentrated $\mathrm{HCl}$ changes the predominant complex to the $\mathrm{CoCl}_{4}^{2-}$ complex, resulting in stronger Faraday rotation at $710 \mathrm{~nm}$ and a strong MCD response at $690 \mathrm{~nm}$. Neutralizing the solution returns the balance to the $\mathrm{Co}\left(\mathrm{H}_{2} \mathrm{O}\right)_{6}^{2+}$ complex, effectively removing the magnetooptical response in this wavelength range. Crucially, subsequent addition of $\mathrm{HCl}$ returns the magneto-optical response to the same magnitude it was before. This indicates that the switching is reversible, an important characteristic for applications. This reversible switching is present in both the MCD and Faraday response. While this simultaneous switching may be desirable in certain applications, it would be interesting in future research to use transitions that are mainly active in either absorption or optical rotation, allowing a separation of both magneto-optical effects.

\section{DISCUSSION AND PERSPECTIVES}

It is possible to change the magneto-optical response of a material by changing its state, such as a change in protonation, oxidation or complexation state. In this work we present measurements of the switching of the 
Faraday effect and MCD in aqueous solutions of cobalt complexes. We have measured the reversible switching of the magneto-optical response of cobalt complexes by acidification of the solution and addition of chlorine ions, and conversely by neutralization of the solution. This easily induced reversible switching opens the possibility of novel devices such as switchable optical isolators. Such devices would allow the variation of optical isolation purely by changing the molecular environment or state.

We have used the acidity of the solution and chlorine concentration to switch the magneto-optical response of cobalt complexes. While interesting as a first step towards tunable magneto-optical response, this is not practical in a device/application context. However, it is possible to extend this methodology to redox reactions. Future work will focus on using redox chemistry to switch magneto-optical characteristics. Cobalt complexes are an excellent initial system to investigate. Cobalt can exist stably in solution in both the $2+$ and $3+$ oxidation state, depending on the solution characteristics. By applying an oxidative potential to a solution of $\mathrm{Co}^{2+}$ it can be oxidized to $\mathrm{Co}^{3+}$. This oxidation can occur purely by applying an electric potential to the present electrodes, and does not require any physical handling. This is ideal for applications such as optical isolators, which cannot be moved during operation without detrimental effects for their application. An extra advantage to an electrochemical approach is that much previous research exists on switching metal-organic compounds and the resulting changes in (nonlinear) optical activity. ${ }^{25}$ These compounds are known to have good nonlinear optical properties and excellent reversibility upon switching. The extended conjugated electrons, coupled to a magnetically active redox active center provide optimal conditions for observing and switching magneto-optical acitivity. Consequently, in the future, their nonlinear magnetic properties could be studied with magnetizationinduced second harmonic generation ${ }^{26}$ a broadly applicable technique ${ }^{27}$ which is sensitive to magnetization down to a single magnetic monolayer ${ }^{28}$ when carefully measured. ${ }^{29}$

\section{ACKNOWLEDGMENTS}

Stefaan Vandendriessche is grateful for the financial support from the FWO-Vlaanderen. We are grateful to the University of Leuven (GOA) for financial support. Ward Brullot received funding from the Agency for Innovation by Science and Technology (IWT) Flanders (research funded by a PhD grant of the Agency for Innovation by Science and Technology (IWT)). We acknowledge useful dicussions with Maarten Bloemen, Ventsislav K. Valev and Maarten Vanbel.

\section{REFERENCES}

[1] Faraday, M., [Experimental Researches in Electricity], vol. III, R. Taylor \& W. Francis (1839-1855).

[2] Takeda, H. and John, S., "Compact optical one-way waveguide isolators for photonic-band-gap microchips," Phys. Rev. A 78, 023804 (2008).

[3] Budker, D., Kimball, D. F., Rochester, S. M., Yashchuk, V. V. and Zolotorev, M., "Sensitive magnetometry based on nonlinear magneto-optical rotation," Phys. Rev. A 62, 043403 (2000).

[4] Bohnert, K., Gabus, P., Kostovic, J. and Brändle, H., "Optical fiber sensors for the electric power industry," Opt. Laser Eng. 43, 511-526 (2005).

[5] Bera, S. C. and Chakraborty, S., "Study of magneto-optic element as a displacement sensor," Measurement 44, 1747-1752 (2011).

[6] Lottermoser, T., Lonkai, T., Amann, U., Hohlwein, D., Ihringer, J. and Fiebig, M., "Magnetic phase control by an electric field.," Nature 430, 541-544 (2004).

[7] Greilich, A., Yakovlev, D. R., Shabaev, A., Efros, A. L., Yugova, I. A., Oulton, R., Stavarache, V., Reuter, D., Wieck, A. and Bayer, M., "Mode locking of electron spin coherences in singly charged quantum dots," Science 313, 341-345 (2006).

[8] Kato, Y. K., Myers, R. C., Gossard, A. C. and Awschalom, D. D., "Current-induced spin polarization in strained semiconductors," Phys. Rev. Lett. 93, 176601 (2004).

[9] Carilli, C. L. and Taylor, G. B., "Cluster magnetic fields," Annu. Rev. Astron. Astr. 40, 319-348 (2002).

[10] Valev, V. K., Wouters, J. and Verbiest, T., "Differential detection for measurements of faraday rotation by means of ac magnetic fields," Eur. J. Phys. 29(5), 1099 (2008). 
[11] Botek, E., Champagne, B., Verbiest, T., Gangopadhyay, P. and Persoons, A., "A joint theoreticalexperimental investigation of the Faraday effect in benzene, toluene, and p-xylene," Chem. Phys. Chem. 7(8), 1654-1656 (2006).

[12] Jaszunski, M., Jørgensen, P., Rizzo, A., Ruud, K. and Helgaker, T., "MCSCF calculations of Verdet constants," Chem. Phys. Lett. 222, 263-266 (1994).

[13] Vandendriessche, S., Valev, V. K. and Verbiest, T., "Faraday rotation and its dispersion in the visible region for saturated organic liquids," Phys. Chem. Chem. Phys. 14, 1860-1864 (2012).

[14] Isai, K., Suwa, M. and Watarai, H., "Pulsed magnetic field faraday imaging of diamagnetic liquids," Anal. Sci. 25(1), 1-3 (2009).

[15] Brullot, W., Reddy, N. K., Wouters, J., Valev, V. K., Goderis, B., Vermant, J. and Verbiest, T., "Versatile ferrofluids based on polyethylene glycol coated iron oxide nanoparticles," J. Magn. Magn. Mater. 324, 1919-1925 (2012).

[16] Tepper, T., Ilievski, F., Ross, C. A., Zaman, T. R., Ram, R. J., Sung, S. Y. and Stadler, B. J. H., "Magnetooptical properties of iron oxide films," J. Appl. Phys. 93(10), 6948-6950 (2003).

[17] Bradley, J. M., Butt, J. N. and Cheesman, M. R., "Electrochemical titrations and reaction time courses monitored in situ by magnetic circular dichroism spectroscopy," Anal. Biochem. 419, 110-116 (2011).

[18] Domingo, N., Williamson, B. E., Gómez-Segura, J., Gerbier, P., Ruiz-Molina, D., Amabilino, D. B., Veciana, J. and Tejada, J., "Magnetism of isolated $\mathrm{Mn}_{12}$ single-molecule magnets detected by magnetic circular dichroism: Observation of spin tunneling with a magneto-optical technique," Phys. Rev. B 69, 052405(2004).

[19] Valev, V. K., Wouters, J. and Verbiest, T., "Precise measurements of Faraday rotation using ac magnetic fields," Am. J. Phys. 76, 626-629 (2008).

[20] Collocott, S. J., "Numerical solution of Kramers-Kronig transforms by a Fourier method," Comput. Phys. Commun. 13, 203-206 (1977).

[21] Denning, R. G. and Spencer, J. A., "Magnetic circular dichroism of the tetrachlorocobaltate ion," Symp. Faraday Soc. 3, 84-91 (1969).

[22] Schooley, D. A., Bunnenberg, E. and Djerassi, C., "Magnetic circular dichroism studies: A preliminary report," P. Natl. Acad. Sci. 53, 579-586 (1965).

[23] Kjaergaard, T., Kristensen, K., Kauczor, J., Jørgensen, P., Coriani, S. and Thorvaldsen, A. J., "Comparison of standard and damped response formulations of magnetic circular dichroism," J. Chem. Phys. 135, 02411216 (2011).

[24] Stephens, P. J., "Dispersion of the Faraday effect in $\mathrm{CoCl}_{4}^{2-}$," J. Chem. Phys. 43, 4444-4446 (1965).

[25] Asselberghs, I., Clays, K., Persoons, A., McDonagh, A. M., Ward, M. D. and McCleverty, J. A., "In situ reversible electrochemical switching of the molecular first hyperpolarizability," Chem. Phys. Lett. 368, 408-411 (2003).

[26] Kirilyuk, A. and Rasing, T., "Magnetization-induced-second-harmonic generation from surfaces and interfaces," J. Opt. Soc. Am. B 22, 148-167 (2005).

[27] Vandendriessche, S., Valev, V. K. and Verbiest, T., "Characterization of magnetization-induced second harmonic generation in iron oxide polymer nanocomposites," Appl. Opt. 51, 209-213 (2012).

[28] Valev, V. K., Kirilyuk, A., Dalla Longa, F., Kohlhepp, J. T., Koopmans, B. and Rasing, T., "Observation of periodic oscillations in magnetization-induced second harmonic generation at the MnCo interface," Phys. Rev. B 75, 012401- (2007).

[29] Valev, V. K., Gruyters, M., Kirilyuk, A., and Rasing, T., "Influence of quadratic contributions in magnetization-induced second harmonic generation studies of magnetization reversal," Phys. Status Solidi B 242(15), 3027-3031 (2005). 